\title{
A fully nonlinear problem with free boundary in the plane
}

\author{
DANiEla De Silva AND ENRICO VALDinoci
}

\begin{abstract}
We prove that bounded solutions to an overdetermined fully nonlinear free boundary problem in the plane are one dimensional. Our proof relies on maximum principle techniques and convexity arguments.
\end{abstract}

Mathematics Subject Classification (2010): 35J60 (primary); 35N25, 35B06 (secondary) .

\section{Introduction}

Let $\phi_{0}, \phi_{1} \in C^{2}(\mathbb{R})$ be such that $\phi_{0}(t)<\phi_{1}(t)$ for any $t \in \mathbb{R}$ and let $\Omega$ be the open set in $\mathbb{R}^{2}$ trapped between the graphs of $\phi_{0}$ and $\phi_{1}$, i.e.

$$
\Omega:=\left\{\left(x_{1}, x_{2}\right) \in \mathbb{R}^{2} \text { such that } \phi_{0}\left(x_{1}\right)<x_{2}<\phi_{1}\left(x_{1}\right)\right\} .
$$

Define

$$
\begin{aligned}
& \mathcal{J}_{0}:=\left\{\left(x_{1}, x_{2}\right) \in \mathbb{R}^{2} \text { such that } x_{2}=\phi_{0}\left(x_{1}\right)\right\}, \\
& \mathcal{J}_{1}:=\left\{\left(x_{1}, x_{2}\right) \in \mathbb{R}^{2} \text { such that } x_{2}=\phi_{1}\left(x_{1}\right)\right\} .
\end{aligned}
$$

Notice that $\partial \Omega=\mathcal{J}_{0} \cup \mathcal{J}_{1}$.

We consider the following problem

$$
\begin{cases}F\left(D^{2} u\right)=0 & \text { in } \Omega, \\ u=0 & \text { on } \mathcal{J}_{0}, \\ u=1 & \text { on } \mathcal{J}_{1},\end{cases}
$$

where $F$ is a uniformly elliptic fully nonlinear operator with ellipticity constants $0<\lambda \leq \Lambda$, and $F(0)=0$ (see [2] for the definition).

In this paper we prove the following symmetry result about bounded solutions to the one-phase free boundary problem associated to (1.1).

EV is supported by FIRB project "Analysis and Beyond" and GNAMPA project "Equazioni non lineari su varietà: proprietà qualitative e classificazione delle soluzioni”.

Received November 13, 2008; accepted in revised form March 20, 2009. 
Theorem 1.1. Let $u \in C^{2}(\Omega) \cap C^{1}(\bar{\Omega})$ be a solution to (1.1). Suppose that there exist $c_{0}, c_{1} \in \mathbb{R}$ such that

$$
\begin{array}{lll}
|\nabla u(x)|=c_{0} & \text { for any } & x \in \mathcal{J}_{0}, \\
|\nabla u(x)|=c_{1} & \text { for any } & x \in \mathcal{J}_{1} .
\end{array}
$$

Assume also that

$$
0<u<1 \text { for any } x \in \Omega .
$$

Then $c_{0}=c_{1}$ and $u$ is a linear function in $\Omega$. In particular $\mathcal{J}_{0}$ and $\mathcal{J}_{1}$ are straight lines.

Without loss of generality, using Hopf lemma and a dilation, we will assume that $c_{1}=1$.

Problem (1.1) may be seen as the fully nonlinear analogue of the ideal fluid jet model of [1]. In this sense, the PDE in (1.1) may be seen as an incompressibility condition and the level sets of $u$ correspond to the stream lines along which the particles of the fluid move, and our assumptions say that the stream lines $\mathcal{J}_{0}$ and $\mathcal{J}_{1}$ which comprise the fluid jet are nice curves.

Also, conditions (1.2) and (1.3) may be seen as pressure conditions on the boundary of the fluid, due to Bernoulli's law.

Differently from the case of [1], here the continuity equation for the incompressible fluid is not given by the standard Laplacian operator but, more generally, by a fully nonlinear elliptic one.

Thus, Theorem 1.1 may be seen as a rigidity result on an fully nonlinear fluid jet model which determines the shape of the fluid (i.e., the domain $\Omega$ ) and the stream lines of the fluid (i.e., the function $u$ ), given some information on the exterior pressure (namely, conditions (1.2) and (1.3)).

The inspiration for Theorem 1.1 came from the work of the first author about rigidity results for fully nonlinear phase transition models [4] and from the work of the second author on overdetermined semilinear problems [6]. We refer to these papers for further motivation and related questions. For different rigidity results for fully nonlinear operators see also [5].

Of course, it would be interesting to know whether the analogue of Theorem 1.1 remains true in higher dimensions.

In order to prove our Theorem 1.1 we show that a given level set $\{u=\sigma\}$ is contained in a strip of arbitrarily small width. The main ingredients in the proof are a Harnack type inequality for the level set $\{u=\sigma\}$ (see Proposition 4.4) and a convexity result which roughly says that if two balls at unit distance from the $\sigma$ level set of $u$ are contained in $\{u<\sigma\}$, then their convex hull is also contained in $\{u<\sigma\}$ (see Proposition 5.1). To obtain these two results, we use the maximum principle together with appropriate radially symmetric barriers.

The rest of the paper is devoted to the proof of Theorem 1.1. Namely, in Section 2 we introduce some notation and tools which will be used throughout the paper. In Section 3, we prove that a solution $u$ as in Theorem 1.1 must satisfy the 
free boundary condition $|\nabla u|=1$ on $\mathcal{J}_{0}$. In Section 4 , we prove a Harnack type inequality for the level sets of $u$ which will be used to show an improvement of flatness for the $\sigma$ level set. In Section 5, we prove the desired convexity property mentioned above and we exhibit the proof of Theorem 1.1.

We conclude our introduction with a remark. In Theorem 1.1 we do not need to consider classical solutions to the free boundary problem. Our result still holds for viscosity solutions (see [3] for the definition) as long as $\mathcal{J}_{0}$ and $\mathcal{J}_{1}$ are continuous graphs.

\section{Main tools}

\subsection{Useful barriers}

In this section we construct suitable super/subsolutions, by modifying the onedimensional solutions to (1.1). The barriers we construct here are inspired by the ones introduced in $[7,9]$ and developed in $[4,10,11]$. We also refer to [8] for related techniques for fully nonlinear equations.

Throughout the paper, constants depending only on the ellipticity constants $\lambda, \Lambda$ will be called universal constants.

Proposition 2.1. Let $\sigma \in[0,1)$. There exist universal constants $C_{i}>0$ such that if $R \geq C_{0}$ and

$$
\begin{aligned}
& g:\left[0, \rho_{1}\right] \rightarrow \mathbb{R}, \quad g\left(\rho_{1}\right)=1, \quad \rho_{1} \leq 2 \\
& g(s):=\sigma+\left(1+\frac{C_{1}}{R}\right) s-\frac{C_{2}}{R} s^{2}
\end{aligned}
$$

then

$$
\beta_{R, \sigma}^{+}(x):=g(|x|-R) \in C^{2}\left(\overline{B_{R_{1}} \backslash B_{R}}\right), \quad R_{1}:=R+\rho_{1}
$$

satisfies

(i) $\beta_{R, \sigma}^{+}$is radially strictly increasing with

$$
\beta_{R, \sigma}^{+}=\sigma \quad \text { on } \partial B_{R}, \quad \beta_{R, \sigma}^{+}=1 \quad \text { on } \partial B_{R_{1}}
$$

(ii) $F\left(D^{2} \beta_{R, \sigma}^{+}\right)<0$ in $B_{R_{1}} \backslash B_{R}$;

(iii) $\left|\nabla \beta_{R, \sigma}^{+}\right| \leq 1+C_{3} / R$ on $\partial B_{R}$ and $\left|\nabla \beta_{R, \sigma}^{+}\right| \geq 1+C_{4} / R$ on $\partial B_{R_{1}}$.

Proof. First let us compute $\rho_{1}$, that is ( $R$ large)

$$
\rho_{1}=\frac{R+C_{1}}{2 C_{2}}\left(1-\sqrt{1-\frac{4 C_{2} R(1-\sigma)}{\left(R+C_{1}\right)^{2}}}\right) .
$$


Since for small $t \geq 0$,

$$
1-\sqrt{1-t} \leq t
$$

we easily obtain that for $R$ large $\rho_{1} \leq 2$. Thus property ( $i$ ) follows immediately by choosing $C_{1}>4 C_{2}$.

To check that (ii) holds, we let $s:=|x|-R$ and we notice that for an orthogonal matrix $O$ we have

$$
\begin{aligned}
F\left(D^{2} \beta_{R, \sigma}^{+}(x)\right) & =F\left(O^{t}\left(\begin{array}{cc}
g^{\prime \prime}(s) & 0 \\
0 & g^{\prime}(s) /|x|
\end{array}\right) O\right) \\
& =F\left(O^{t}\left(\begin{array}{cc}
-2 C_{2} / R & 0 \\
0 & {\left[\left(1+C_{1} / R\right)-2 C_{2} s / R\right] /|x|}
\end{array}\right) O\right) \\
& \leq \frac{\Lambda}{|x|}\left|1+\frac{C_{1}}{R}-2 \frac{C_{2}}{R} s\right|-\frac{2 \lambda C_{2}}{R} \\
& \leq \frac{2 \Lambda}{R}-\frac{2 \lambda C_{2}}{R}<0
\end{aligned}
$$

for $|x|>R$, large $R$ and $C_{2}>\Lambda / \lambda$.

Property (iii) also follows immediately, since

$$
g^{\prime}(0)=1+C_{1} / R \quad \text { and } \quad g^{\prime}\left(\rho_{1}\right)=1+C_{1} / R-2 C_{2} \rho_{1} / R \geq 1+C_{2} / R
$$

as long as $C_{1} \geq 5 C_{2}$.

Similar arguments also give the following proposition.

Proposition 2.2. Let $\sigma \in[0,1)$. There exist universal constants $\tilde{C}_{i}>0$ such that if $R \geq \tilde{C}_{0}$ and

$$
\begin{aligned}
& \tilde{g}:\left[\tilde{\rho}_{1}, 0\right] \rightarrow \mathbb{R}, \quad \tilde{g}\left(\tilde{\rho}_{1}\right)=1, \quad \tilde{\rho}_{1} \geq-3 \\
& \tilde{g}(s):=\sigma-\left(1-\frac{\tilde{C}_{1}}{R}\right) s-\frac{\tilde{C}_{2}}{R} s^{2}
\end{aligned}
$$

then

$$
\beta_{R, \sigma}^{-}(x):=\tilde{g}(|x|-R) \in C^{2}\left(\overline{B_{R} \backslash B_{\tilde{R}_{1}}}\right), \quad \tilde{R}_{1}:=R+\tilde{\rho}_{1}
$$

satisfies

(i) $\beta_{R, \sigma}^{-}$is radially strictly decreasing with

$$
\beta_{R, \sigma}^{-}=\sigma \quad \text { on } \partial B_{R}, \quad \beta_{R, \sigma}^{-}=1 \quad \text { on } \partial B_{\tilde{R}_{1}} ;
$$


(ii) $F\left(D^{2} \beta_{R, \sigma}^{-}\right)>0$ in $B_{R} \backslash B_{\tilde{R}_{1}}$;

(iii) $\left|\nabla \beta_{R, \sigma}^{-}\right| \geq 1-\tilde{C}_{3} / R$ on $\partial B_{R}$ and $\left|\nabla \beta_{R, \sigma}^{-}\right| \leq 1-\tilde{C}_{4} / R$ on $\partial B_{\tilde{R}_{1}}$.

When $\sigma \in(0,1)$, it is useful to extend the barrier $\beta_{R, \sigma}^{+}$also to values below $\sigma$, as in the following result.

Proposition 2.3. Let $\sigma \in(0,1)$. There exist universal constants $\bar{C}_{i}>0$ such that if $R \geq \bar{C}_{0}$ and

$$
\begin{gathered}
\bar{g}:\left[\rho_{0}, \rho_{1}\right] \subset[-2,2] \rightarrow \mathbb{R}, \quad \bar{g}\left(\rho_{0}\right)=0, \quad \bar{g}\left(\rho_{1}\right)=1, \quad \rho_{0}<0<\rho_{1} \\
\bar{g}(s):=\sigma+\left(1+\operatorname{sign}(s) \frac{\bar{C}_{1}}{R}\right) s-\frac{\bar{C}_{2}}{R} s^{2}
\end{gathered}
$$

then

$$
\begin{aligned}
\bar{\beta}_{R, \sigma}(x) & :=\bar{g}(|x|-R) \in C^{2}\left(\left(\overline{B_{R_{1}} \backslash B_{R_{0}}}\right) \backslash \partial B_{R}\right), \\
R_{1} & :=R+\rho_{1}, \quad R_{0}:=R+\rho_{0},
\end{aligned}
$$

satisfies

(i) $\bar{\beta}_{R, \sigma}$ is radially strictly increasing with

$$
\bar{\beta}_{R, \sigma}=0 \quad \text { on } \partial B_{R_{0}}, \quad \bar{\beta}_{R, \sigma}=\sigma \quad \text { on } \partial B_{R}, \quad \bar{\beta}_{R, \sigma}=1 \quad \text { on } \partial B_{R_{1}} ;
$$

(ii) $F\left(D^{2} \bar{\beta}_{R, \sigma}\right)<0$ in $\left(B_{R_{1}} \backslash B_{R_{0}}\right) \backslash \partial B_{R}$;

(iii) $\left|\nabla \bar{\beta}_{R, \sigma}\right| \leq 1-\bar{C}_{3} / R$ on $\partial B_{R_{0}}$ and $\left|\nabla \bar{\beta}_{R, \sigma}\right| \geq 1+\bar{C}_{4} / R$ on $\partial B_{R_{1}}$.

Proof. In view of Proposition 2.1, we only need to focus on the case $s \in\left[\rho_{0}, 0\right]$ and check that the properties above hold by possibly choosing the constants $\bar{C}_{1}, \bar{C}_{2}$ larger than $C_{1}, C_{2}$. The proof follows from similar computations as in the proof of Proposition 2.1. We sketch it for completeness.

Property (i) is obvious, as long as $R$ is large.

Again, one can easily compute that

$$
\rho_{0}=\frac{R+\bar{C}_{1}}{2 C_{2}}\left(1-\sqrt{1+4 \frac{\bar{C}_{2} R \sigma}{\left(R+\bar{C}_{1}\right)^{2}}}\right) .
$$

Since for $t \geq 0$ we have

$$
1-\sqrt{1+t} \geq-t
$$

we can estimate that

$$
\rho_{0} \geq-2
$$


To check that (ii) holds, again we let $s:=|x|-R$ then, for an orthogonal matrix $O$, we have

$$
\begin{aligned}
F\left(D^{2} \bar{\beta}_{R, \sigma}(x)\right) & =F\left(O^{t}\left(\begin{array}{cc}
\bar{g}^{\prime \prime}(s) & 0 \\
0 & \bar{g}^{\prime}(s) /|x|
\end{array}\right) O\right) \\
& \leq \frac{\Lambda}{|x|}\left|1-\frac{\bar{C}_{1}}{R}-2 \frac{\bar{C}_{2}}{R} s\right|-\frac{2 \lambda \bar{C}_{2}}{R} \\
& \leq \frac{2 \Lambda}{R}-\frac{2 \lambda \bar{C}_{2}}{R}<0
\end{aligned}
$$

for $|x|>R_{0}$, large $R$, and $\bar{C}_{1}>4 \bar{C}_{2}, \bar{C}_{2}>\Lambda / \lambda$.

Property (iii) also follows immediately, since

$$
\bar{g}^{\prime}\left(\rho_{0}\right)=1-\bar{C}_{1} / R-2 \bar{C}_{2} \rho_{0} / R \leq 1-\bar{C}_{2} / R
$$

as long as $\bar{C}_{1} \geq 5 \bar{C}_{2}$.

Clearly, using similar arguments, also the barrier $\beta_{R, \sigma}^{-}$can be extended below $\sigma$ to a barrier $\underline{\beta}_{R, \sigma}$. We omit its precise definition, since we do not explicitly need it here.

From now on we extend $\bar{\beta}_{R, \sigma}$ to be 0 in $B_{R_{0}}$. Also, sometimes we think that $\bar{\beta}_{R, \sigma}$ is extended to 1 outside of $B_{R_{1}}$. This will be clear from the context.

Remark 2.4. We point out that all the $\rho_{i}$ 's introduced in Propositions 2.1, 2.2 and 2.3 are bounded by universal constants - this will be very important, for instance, in Lemma 4.3 below.

Remark 2.5. Notice that, since $-2 \leq \rho_{0}<\rho_{1} \leq 2$ one has

$$
|\bar{g}(s)-(s+\sigma)| \leq \frac{\bar{C}_{5}}{R}, \quad s \in\left[\rho_{0}, \rho_{1}\right]
$$

In particular, evaluating (2.1) at $s=\rho_{0}$ and at $s=\rho_{1}$, we see that

$$
\begin{aligned}
& R-\sigma-\frac{\bar{C}_{5}}{R} \leq R_{0} \leq R-\sigma+\frac{\bar{C}_{5}}{R}, \\
& R+(1-\sigma)-\frac{\bar{C}_{5}}{R} \leq R_{1} \leq R+(1-\sigma)+\frac{\bar{C}_{5}}{R} .
\end{aligned}
$$

Define $T_{0}^{1}$ to be the truncation at levels 0 and 1, i.e.

$$
T_{0}^{1}(t):= \begin{cases}0 & \text { if } t<0 \\ t & \text { if } 0 \leq t \leq 1 \\ 1 & \text { if } t>1\end{cases}
$$


Then from (2.1) it follows that

$$
T_{0}^{1}\left(s+\sigma+C_{5} / R\right) \geq \bar{g}(s) \quad \text { on }\left[\rho_{0}, \rho_{1}\right]
$$

and hence

$$
T_{0}^{1}\left(|x|-R+\sigma+C_{5} / R\right) \geq \bar{\beta}_{R, \sigma}(x) \text { in } \mathbb{R}^{2}
$$

\subsection{Sliding method}

In what follows, the barriers constructed above and one-dimensional solutions to (1.1) will be used as comparison functions in what we call the sliding method. For the sake of clarity, we fix here some notation and terminology which we will use throughout the rest of the paper.

Let $u$ be a solution to (1.1)-(1.4). From now on any such $u$ is extended to be 0 below $\mathcal{J}_{0}$ and 1 above $\mathcal{J}_{1}$ (when not specified "below" and "above" are intended with respect to the $\left.e_{2} \operatorname{direction}^{1}\right)$.

Given a continuous function $v: K \rightarrow \mathbb{R}, K$ compact, we set

$$
v_{t}(x):=v\left(x-t e_{2}\right), \quad t \in \mathbb{R} .
$$

We say that $v$ is above $u$ if $v \geq u$ in $K$. Also, we say that $v$ touches $u$ from above if $v$ is above $u$ and $v_{t}$ is not above $u$ for any $t>0$. Clearly, this implies that $u\left(x_{0}\right)=v\left(x_{0}\right)$ at some $x_{0} \in K \cap \bar{\Omega}$ which we call a contact point.

The sliding method can be roughly described as follows. Assume that for some $t_{0}$ we have that $v_{t_{0}} \geq u$. Then by continuity, there exists the smallest $\bar{t} \geq t_{0}$ such that $v_{\bar{t}}$ touches $u$ from above. If $v \neq u$ is a supersolution to our equation, then we can apply the maximum principle to conclude that contact points are in $\partial K$. If we also know for example that $|\nabla v| \leq c_{0}$ (respectively $|\nabla v| \geq 1$ ) on $\{v=0\}$ (respectively on $\{v=1\})$ then we can conclude from Hopf lemma that no contact points occur on $\{v=0\}$ (respectively on $\{v=1\}$ ) unless $v=u$.

This method will be a key tool in all our proofs.

\section{Determining $c_{0}$}

The purpose of this section is to determine $c_{0}$, that is the exterior pressure of the fluid on $\mathcal{J}_{0}$, by knowing the pressure on $\mathcal{J}_{1}$. Precisely, we prove the following proposition.

Proposition 3.1. Let $u \in C^{2}(\Omega) \cap C^{1}(\bar{\Omega})$ be a solution to (1.1)-(1.4). Then $c_{0}=1$.

Proof. We apply the sliding method with the comparison function $v=\beta_{R, 0}^{+}$in Proposition 2.1 (we use the notation in that proposition).

${ }^{1}$ Here and below, the term direction denotes a vector of unit length. 
Since $u=0$ below $\mathcal{J}_{0}$ and $v \geq 0$ in $B_{R_{1}}$ there exists a $t_{0}<0$ such that $v_{t_{0}}$ is above $u$. Let $\bar{t}$ be the smallest $t \geq t_{0}$ such that $v_{t}$ touches $u$ from above.

According to Proposition 2.1(ii), $v_{\bar{t}}$ is a strict supersolution in $B_{R_{1}} \backslash B_{R}$, thus by the comparison principle if $\bar{x}$ is a contact point then

$$
\bar{x} \in \partial\left(B_{R_{1}}(0, \bar{t}) \backslash B_{R}(0, \bar{t})\right) .
$$

We now show that

$$
\bar{x} \notin \partial B_{R_{1}}(0, \bar{t}) .
$$

Indeed suppose by contradiction that $\bar{x} \in \partial B_{R_{1}}(0, \bar{t})$. Then, by Proposition 2.1(i), we have $v_{\bar{t}}(\bar{x})=1$ and so since $0<u<1$ in $\Omega$,

$$
\bar{x} \in \mathcal{J}_{1} .
$$

Also, if $v$ is the exterior normal of $\partial B_{R_{1}}(0, \bar{t})$ we get

$$
\partial_{\nu} v_{\bar{t}}(\bar{x}) \leq \partial_{\nu} u(\bar{x}) .
$$

Then, the inequality above together with Proposition 2.1(i), (1.3), (3.3), give

$$
\left|\nabla v_{\bar{t}}(\bar{x})\right|=\partial_{\nu} v_{\bar{t}}(\bar{x}) \leq 1 .
$$

Since $\bar{x} \in \partial B_{R_{1}}(0, \bar{t})$, this inequality contradicts Proposition 2.1(iii), and so (3.2) is proved.

Therefore by (3.1) and (3.2) we obtain that

$$
\bar{x} \in \partial B_{R}(0, \bar{t}) .
$$

Thus, by Proposition 2.1(i), we have that $\bar{x} \in \mathcal{J}_{0}$ and so, by (1.2), (3.4) and Proposition 2.1(i), (iii), we conclude that

$$
c_{0}=|\nabla u(\bar{x})| \leq\left|\nabla v_{\bar{t}}(\bar{x})\right| \leq 1+\frac{C_{3}}{R} .
$$

Now we perform a (upside-down) sliding argument with comparison function $w=$ $\beta_{R, 0}^{-}$(recall Proposition 2.2).

Since $u=1$ above $\mathcal{J}_{1}$, there exists $t_{0}>0$ such that $u$ is above $w_{t_{0}}$. Let $t$ be the largest $t \leq t_{0}$ such that $u$ touches $w_{t}$ from above.

According to Proposition 2.2(ii) $w_{\underline{t}}$ is a strict subsolution thus, by the comparison principle, we know that if $\underline{x}$ is a contact point then

$$
\underline{x} \in \partial\left(B_{R}(0, \underline{t}) \backslash B_{\tilde{R}_{1}}(0, \underline{t})\right) .
$$

As before, we deduce that

$$
\underline{x} \notin \partial B_{\tilde{R}_{1}}(0, \underline{t}) .
$$


Indeed, if (3.7) were false, we would have that $u(\underline{x})=1$ thanks to Proposition 2.2(i). Accordingly, from (1.3) and Proposition 2.2(iii),

$$
1=|\nabla u(\underline{x})| \leq\left|\nabla w_{\underline{t}}(\underline{x})\right| \leq 1-\frac{\tilde{C}_{3}}{R} .
$$

This contradiction proves (3.7).

Then, from (3.6) and (3.7), we deduce that $\underline{x} \in \partial B_{R}(0, \underline{t})$ and so, from Proposition 2.2(i) and (1.4), that $\underline{x} \in \mathcal{J}_{0}$.

Finally, (1.2) and Proposition 2.2(i), (iii) give that

$$
c_{0}=|\nabla u(\underline{x})| \geq\left|\nabla w_{\underline{t}}(\underline{x})\right| \geq 1-\frac{\tilde{C}_{4}}{R} .
$$

This and (3.5) imply that $c_{0}=1$, by taking $R$ arbitrarily large.

\section{Level set analysis}

In this section we wish to prove a Harnack type inequality for the level sets of a solution $u$ to our problem. The techniques we use here and in the next section have been inspired by [4].

From now on we denote by $u$ a solution to

$$
\begin{cases}F\left(D^{2} u\right)=0 & \text { in } \Omega \\ u=0,|\nabla u|=1 & \text { on } \mathcal{J}_{0} \\ u=1,|\nabla u|=1 & \text { on } \mathcal{J}_{1}\end{cases}
$$

with $0<u<1$ in $\Omega$. As usually $u$ is extended to be 0 below $\mathcal{J}_{0}$ and 1 above $\mathcal{J}_{1}$.

As in the case of the one-phase problem in [3], one obtains that $u$ is Lipschitz continuous with universal bound.

Lemma 4.1. $u$ is uniformly Lipschitz continuous, i.e

$$
\|\nabla u\|_{L^{\infty}} \leq K
$$

with $K$ universal constant.

Proof. Let $x_{0} \in \Omega$, and $d=\min \left\{\operatorname{dist}\left(x_{0}, \mathcal{J}_{0}\right)\right.$, $\left.\operatorname{dist}\left(x_{0}, \mathcal{J}_{1}\right)\right\}$. Assume without loss of generality that $d=\operatorname{dist}\left(x_{0}, \mathcal{J}_{0}\right)$. We wish to prove that

$$
\left|\nabla u\left(x_{0}\right)\right| \leq K
$$

Let

$$
v(x)=\frac{1}{d} u\left(x_{0}+d x\right)
$$


be the rescale of $u$ in $B_{d}\left(x_{0}\right)$. Then $v \geq 0$ solves a uniformly elliptic equation

$$
G\left(D^{2} v\right)=0 \text { in } B_{1}(0),
$$

with $G(M)=d F(M / d)$ having the same ellipticity constants as $F$, and $G(0)=0$. Hence, by Harnack's inequality (see [2])

$$
v \geq c v(0) \text { in } B_{1 / 2}(0) .
$$

Let us choose $\beta<0$ such that, the radially symmetric function

$$
g(x)=\frac{c v(0)}{2^{-\beta}-1}\left(|x|^{\beta}-1\right)
$$

satisfies $G\left(D^{2} g\right) \geq 0$ in the annulus $B_{1} \backslash \overline{B_{1 / 2}}, g=0$ on $\partial B_{1}$ and $g=c v(0) \leq v$ on $\partial B_{1 / 2}$, due to (4.3).

Then, by the maximum principle

$$
v \geq g \text { in } B_{1} \backslash \overline{B_{1 / 2}} .
$$

Now, let $x_{1} \in \partial B_{1}(0)$ be such that $v\left(x_{1}\right)=0$. Then, since $\nabla v(x)=\nabla u\left(x_{0}+d x\right)$, and $u$ solves (4.1), we have $\left|\nabla v\left(x_{1}\right)\right|=1$. Let $v$ be the inward normal to $\partial B_{1}$ at $x_{1}$.

Then,

$$
1=\left|\nabla v\left(x_{1}\right)\right| \geq v_{v}\left(x_{1}\right) \geq g_{v}\left(x_{1}\right) \geq C v(0) .
$$

Using Harnack's inequality we conclude that

$$
\|v\|_{L^{\infty}\left(B_{1 / 2}\right)} \leq C .
$$

Also, by the $C^{1, \alpha}$ estimate for equation (4.2) (see [2]), we have that

$$
\|v\|_{C^{1, \alpha}\left(B_{1 / 4}\right)} \leq C\|v\|_{L^{\infty}\left(B_{1 / 2}\right)} .
$$

Therefore, from (4.4) and (4.5), we obtain that $|\nabla v(0)|=\left|\nabla u\left(x_{0}\right)\right|$ is bounded by a universal constant.

Now we fix a $\sigma \in(0,1)$ and we proceed to analyze the properties of the correspondent level set of $u$, that is $\{u=\sigma\}$.

We start with a definition and an elementary lemma and then we state and prove the desired Harnack type inequality. In the next section, towards proving our Theorem 1.1, we will show that $\{u=\sigma\}$ is flat enough, i.e. it is contained in a strip. Then, Harnack inequality will be used to prove an improvement of flatness for $\{u=\sigma\}$ which implies the result of Theorem 1.1.

Definition 4.2. We define $A_{R, \sigma}$ to be the set of all $x$ 's such that

$$
B_{R}(x) \subseteq\{u<\sigma\} \quad \text { and } \quad \overline{B_{R}}\left(x+t e_{2}\right) \subset\{u<\sigma\} \text { for all } t<0 .
$$


In other words, $A_{R, \sigma}$ consists of the centers of the balls of radius $R$ whose translates in the direction $-e_{2}$ are compactly included in $\{u<\sigma\}$.

Lemma 4.3. Assume that $x_{*} \in A_{R, \sigma}, R$ large, then

(i) $u(x) \leq \bar{\beta}_{R, \sigma}\left(x-x_{*}\right)$;

(ii) If $x_{0} \in \partial B_{R}\left(x_{*}\right) \cap\{u=\sigma\}$, then $\mathcal{J}_{1} \cap B_{K}\left(x_{0}\right) \neq \emptyset$ for some universal constant $K$.

Proof. Claim (i) follows easily by applying the sliding method with the comparison function $\bar{\beta}_{R, \sigma}\left(x-x_{*}\right)$, where the notation of Proposition 2.3 is used.

The argument is similar to the proof of Proposition 3.1. Touching along $\mathcal{J}_{0}, \mathcal{J}_{1}$ is excluded by Proposition 2.3(iii) and contact points always occur on $\{u=\sigma\}$.

In order to prove (ii), we observe that in Proposition 2.3 we can take $\sigma=1$ and $\rho_{1}=0$. Then, for $R$ large we have that $\bar{\beta}_{R, 1}$ is a strict supersolution in $B_{R} \backslash B_{R_{0}}$ and it satisfies the strict free boundary condition $\left|\nabla \bar{\beta}_{R, 1}\right|<1$ on $\partial B_{R_{0}}$.

Now we fix $K$ large and apply the sliding method in the direction $v$ of $x_{0}-x_{*}$ with comparison function $v(x)=\bar{\beta}_{K / 2,1}\left(x-x_{*}\right)$. This means that the translates $v_{t}(x)=v(x-t v)$ are in the $v$ direction.

According to (i), $u=0$ in $B_{K / 2}\left(x_{*}\right)(R>K)$, thus $v$ is above $u$ in such ball. Let $\bar{t}$ be the smallest $t \geq 0$ such that $v_{t}$ touches $u$ from above. Since $v_{\bar{t}}$ is a strict supersolution and it satisfies the strict free boundary condition $\left|\nabla v_{\bar{t}}\right|<1$ on its zero level set we conclude that touching points can only occur on

$$
\partial B_{K / 2}\left(x_{*}+\bar{t} v\right) \cap \mathcal{J}_{1} \neq \emptyset \text {. }
$$

In particular this implies,

$$
\bar{t} \geq R-K / 2 .
$$

On the other hand, $\bar{t} \leq R$ since $0=v_{R}\left(x_{0}\right)<u\left(x_{0}\right)=\sigma$. The bounds on $\bar{t}$ imply that $x_{0} \in B_{K / 2}\left(x_{*}+\overline{\bar{t}} v\right)$ which together with (4.6) yield the desired result.

Lemma 4.4 (Harnack Inequality). Assume that

$$
\partial_{x_{2}} u \geq 0 \text { in } \Omega \text {. }
$$

If $x_{*}=-R v \in A_{R, \sigma}$ and

$$
0 \in\{u=\sigma\} \cap \partial B_{R}\left(x_{*}\right), \quad \nu_{2}>0,
$$

then, for any $M>0$ there exist $\bar{R}, \bar{C}$ depending on $\sigma, \nu_{2}, M, \lambda, \Lambda$ such that if $R \geq \bar{R}$ then

$$
B_{M-\frac{\bar{C}}{R}}(M v) \subset\{u>\sigma\}
$$


Proof. Let

$$
w(x)=T_{0}^{1}\left(x \cdot v+\sigma+\frac{C_{1}}{R}\right),
$$

with $C_{1}$ to be chosen later (see (2.3) for the definition of $T_{0}^{1}$ ).

Notice that $F\left(D^{2} w\right)=0$ and $|\nabla w|=1$ in $\{0<w<1\}$.

First, we wish to show that if $R$ is large enough

$$
w(x) \geq \bar{\beta}_{R, \sigma}\left(x-x_{*}\right) \quad \text { for any } x \in B_{\frac{20 M}{v_{2}}}(0) .
$$

Since $T_{0}^{1}$ is non-decreasing, in view of (2.4) it suffices to show that

$$
x \cdot v+\sigma+C_{1} / R \geq\left|x-x_{*}\right|-R+\sigma+\bar{C}_{5} / R \quad \text { in } B_{\frac{20 M}{\nu_{2}}}(0) .
$$

Thus we need to prove that

$$
x \cdot v+R+C_{2} / R \geq\left|x-x_{*}\right| \text { for any } x \in B_{\frac{20 M}{v_{2}}}(0),
$$

for some $C_{2}$ large.

For this we write

$$
x=: a v+b v^{\perp} \in B_{\frac{20 M}{v_{2}}}(0), \quad|a|,|b| \leq 20 M / \nu_{2} .
$$

Since $x_{*}=-R v,(4.10)$ may be written as

$$
a+R+C_{2} / R \geq\left|(a+R) v+b v^{\perp}\right|
$$

or, equivalently,

$$
\left(a+R+C_{2} / R\right)^{2} \geq(a+R)^{2}+b^{2} .
$$

Hence it is enough to prove that

$$
(1+2 a / R) C_{2} \geq b^{2} .
$$

Since $|a|,|b| \leq 20 M / \nu_{2}$ this inequality holds for $R$ and $C_{2}$ large depending on $M / \nu_{2}$. Thus (4.9) is proved.

As a consequence, in view of Lemma 4.3(i), we obtain that

$$
w \geq u \quad \text { in } B_{\frac{20 M}{\nu_{2}}}(0)
$$

Now, according to Lemma $4.1 u$ is uniformly Lipschitz with universal Lipschitz constant $K$. Thus, if

$$
u(x) \in I:=[\sigma / 2,1 / 2+\sigma / 2]
$$


then there exists a constant $c$ depending on $K, \sigma$ such that

$$
B_{c}(x) \subset\{0<u<1\} .
$$

Clearly, the same statement holds also for $w$, that is

$$
B_{c}(x) \subset\{0<w<1\}
$$

and so $F\left(D^{2} w\right)=0$ in $B_{c}(x)$, as long as $w(x) \in I$.

In particular, we observe that $u(0), w(0) \in I$, so

$$
F\left(D^{2} w\right)=F\left(D^{2} u\right)=0 \quad \text { in } B_{c}(0) .
$$

Also, by (4.8),

$$
w(0)-u(0)=C_{1} / R .
$$

So, in view of (4.11) we can apply the Harnack inequality in $B_{c}(0)$ and obtain

$$
w-u \leq C^{\prime} / R \quad \text { in } B_{c / 2}(0) .
$$

Now, we observe that $\{w=\sigma\}$ is the line $\left\{x \cdot v=-C_{1} / R\right\}$, due to (4.8), and therefore we pick $x_{1} \in B_{c / 2}(0) \cap\{w=\sigma\}$ (of course, this is possible since $R$ is large enough).

Then,

$$
0 \leq w\left(x_{1}\right)-u\left(x_{1}\right) \leq C^{\prime} / R
$$

and $u\left(x_{1}\right), w\left(x_{1}\right) \in I$. Arguing as above, we conclude that

$$
w-u \leq C^{\prime \prime} / R \text { in } B_{c / 2}\left(x_{1}\right) .
$$

Now we can pick $x_{2} \in B_{c / 2}\left(x_{1}\right) \cap\{w=\sigma\}$ and iterate this argument: after a finite number, say $k_{o}$, of these iterations, we cover $\{w=\sigma\} \cap B_{10 M / v_{2}}(0)$ with the balls $B_{c / 2}\left(x_{1}\right), B_{c / 2}\left(x_{2}\right), \ldots, B_{c / 2}\left(x_{k_{o}}\right)$, which have the property that

$$
w-u \leq C(j) / R \text { in } B_{c / 2}\left(x_{j}\right), \text { for } j=1, \ldots, k_{o} .
$$

Since the above $k_{o}$ is finite and it only depends on $\sigma, M / \nu_{2}$, we conclude that in a $c / 4$ neighborhood $N$ of $\{w=\sigma\} \cap B_{10 M / v_{2}}(0)$ we have that

$$
0 \leq w-u \leq C / R
$$

with $C$ depending also on $\sigma, M / \nu_{2}$.

If

$$
x \in L:=\{x \cdot v=C / R\} \cap B_{10 M / v_{2}}(0)
$$

then

$$
w(x)=\sigma+\frac{C+C_{1}}{R} .
$$


Also, $x \in N$ if $R$ is large, and so from (4.13) we get

$$
u(x)>\sigma, \quad \text { for any } x \in L .
$$

Therefore, since $u$ is monotone in the vertical direction, (4.7) will follow if we show that

$$
B_{M-\frac{\bar{C}}{R}}(M v) \text { is above } L \text { in the vertical direction. }
$$

For this, we choose $\bar{C}:=C$ and we observe that (4.15) is proved if we show that

$$
\begin{aligned}
& \forall x \text { such that }|x-M \nu| \leq M-C / R, \\
& \exists \tilde{x} \in L \text { such that } x-\tilde{x}=t e_{2}, t \geq 0 .
\end{aligned}
$$

Hence, in order to prove (4.16) (and so to complete the proof of the lemma), given $x$ as in (4.16), we let

$$
\tilde{x}:=\left(x_{1}, \frac{1}{v_{2}}\left(\frac{C}{R}-x_{1} v_{1}\right)\right) .
$$

Clearly $\tilde{x} \cdot v=C / R$.

Moreover, since $|x|<2 M$, then $\left|\tilde{x}_{1}\right|<2 M / \nu_{2}$, and $\left|\tilde{x}_{2}\right|<3 M / \nu_{2}$ which implies that $\tilde{x} \in B_{10 M / v_{2}}(0)$, hence

$$
\tilde{x} \in L .
$$

Furthermore,

$$
\left(x-\frac{C}{R} v\right) \cdot v \geq 0
$$

which immediately implies that $x_{2} \geq \tilde{x}_{2}$. This and (4.17) prove (4.16).

\section{Convexity arguments and proof of Theorem 1.1}

The purpose of this section is to exhibit the proof of Theorem 1.1. As mentioned in the previous section, our aim is first to show that $\{u=\sigma\}$ is flat enough, that is, it lies inside a strip.

We start with two propositions which will be the key tools to achieve our goal. Roughly, the first proposition gives the convexity of the set $\{u<\sigma\}$ while the second one gives the convexity of $\{u>\sigma\}$. However, their statements are different since the first one requires the existence of two balls contained in $\{u<\sigma\}$ while the second one requires the existence of two points on or above $\mathcal{J}_{1}$.

Proposition 5.1. Assume $y_{i} \in A_{R, \sigma}, i=1,2$. Then the convex envelope generated by the balls $B_{R-C / R}\left(y_{i}\right), i=1,2$ is included in $\{u<\sigma\}$, for $R$ large and $C$ universal. 
Proof. Let $l$ be the common tangent line from above to $B_{R-C / R}\left(y_{i}\right), i=1,2$ and denote by $x_{i}, i=1,2$ the respective points of tangency.

Let $v$ be the one-dimensional linear solution to $F\left(D^{2} v\right)=0$ in $\{0<v<1\}$ with $|\nabla v|=1$ which equals $\sigma$ on the line $l$, and denote by $l_{0}$ and $l_{1}$ the 0 and the 1 level set of $v$ respectively.

Call $z_{i}, \xi_{i}$ the intersection points of $l_{i}$ and the lines passing through $y_{1}, x_{1}$ and $y_{2}, x_{2}$ respectively $(i=0,1)$. Also, let $D$ be the open rectangle with vertices at $z_{i}, \xi_{i}, i=0,1$.

The segment joining any two points $\eta$ and $\zeta$ which contains $\zeta$ but not $\eta$ will be denoted by $(\eta, \zeta]$. If it contains both $\eta$ and $\zeta$, we write $[\eta, \zeta]$.

In this notation, we claim that

$v>u$ on $S:=\left(z_{0}, z_{1}\right] \cup\left(\xi_{0}, \xi_{1}\right]$, and $z_{0}$ and $\xi_{0}$ are strictly below $\mathcal{J}_{0}$.

We assume for the moment that the claim in (5.1) holds, and we apply the sliding method.

Since $u=0$ below $\mathcal{J}_{0}$ there exists a $t_{0}<0$ such that $v_{t}$ is above $u$ for all $t \leq t_{0}$. Let $\bar{t}$ be the smallest $t \geq t_{0}$ such that $v_{t}$ touches $u$ from above. Assume by contradiction that $\bar{t} \leq 0$.

Then, since $y_{i}+t e_{2} \in A_{R, \sigma}$ for all $t<0$, clearly the claim in (5.1) holds also for $v_{\bar{t}}$, that is

$$
\begin{gathered}
v_{\bar{t}}>u \text { on } S+\bar{t} e_{2} \text { and } \\
z_{\bar{t}}:=z_{0}+\bar{t} e_{2}, \xi_{\bar{t}}:=\xi_{0}+\bar{t} e_{2} \quad \text { are strictly below } \mathcal{J}_{0} .
\end{gathered}
$$

Thus, no contact points can occur in $D_{\bar{t}}:=D+\bar{t} e_{2}$, otherwise $u$ and $v_{\bar{t}}$ coincide and (5.2) is contradicted.

Also, in view of Proposition 3.1 and Hopf lemma, contact points cannot occur on the 0 and 1 level set of $v_{\bar{t}}$ except at the vertices of $D_{\bar{t}}$. Hence, using again (5.2) we conclude that

$$
v_{\bar{t}}>u \text { in } \bar{D}_{\bar{t}} \backslash\left[z_{\bar{t}}, \xi_{\bar{t}}\right] \text { and }\left[z_{\bar{t}}, \xi_{\bar{t}}\right] \Subset\{u=0\} .
$$

This implies that for a small $\varepsilon>0$ the translate $v_{\bar{t}+\varepsilon}$ is above $u$ which contradicts the definition of $\bar{t}$. Thus, $\bar{t}>0$ and by the arguments above

$$
v_{t}>u \text { in } \bar{D}_{t} \backslash\left[z_{t}, \xi_{t}\right] \text { for all } t \leq 0 \text {. }
$$

In particular, since we have chosen $v$ so that its $\sigma$ level set coincides with $l$, we obtain that $u<\sigma$ below $\left[x_{1}, x_{2}\right]$. This gives the result stated in the proposition.

We are left with the proof of the claim in (5.1).

For this, let us show that

$$
v>u \text { on }\left(z_{0}, z_{1}\right] \text { and } z_{0} \text { is strictly below } \mathcal{J}_{0} \text {. }
$$

Call $v=\left(x_{1}-y_{1}\right) /\left|x_{1}-y_{1}\right|$ and observe that

$$
z_{0}=y_{1}+\left(R-\frac{C}{R}-\sigma\right) \nu, \quad z_{1}=y_{1}+\left(R-\frac{C}{R}+1-\sigma\right) \nu .
$$


Hence, making use of (2.2) and taking $C$ large enough, we have that

$$
\left[z_{0}, z_{1}\right] \subset B_{R_{1}}\left(y_{1}\right)
$$

and

$$
z_{0} \in B_{R_{0}} \text {. }
$$

Also, we know from Lemma 4.3(i) that

$$
\bar{\beta}_{R, \sigma}\left(x-y_{1}\right) \geq u(x) \quad \text { in } B_{R_{1}}\left(y_{1}\right) .
$$

Thus, (5.5) and (5.6) say that (5.3) will follow if we show that

$$
v(x)>\bar{\beta}_{R, \sigma}\left(x-y_{1}\right) \quad \text { on }\left(B_{R_{1}}\left(y_{1}\right) \backslash B_{R_{0}}\left(y_{1}\right)\right) \cap\left(z_{0}, z_{1}\right] .
$$

For this, we observe that, by (5.4),

$$
v(x)=\sigma+s+\frac{C}{R} \quad \text { if } x=y_{1}+(s+R) v .
$$

So, by applying (5.4) and (2.1), if $C$ suitably large, we see that on $\left(z_{0}, z_{1}\right]$

$$
\begin{aligned}
v(x)-\bar{\beta}_{R, \sigma}\left(x-y_{1}\right) & =\sigma+s+\frac{C}{R}-\bar{g}\left(\left|x-y_{1}\right|-R\right) \\
& =\sigma+s+\frac{C}{R}-\bar{g}(s)>0 .
\end{aligned}
$$

This proves (5.7) and so (5.3).

By replacing $z_{i}$ with $\xi_{i}$ in the proof of (5.3), one completes the proof of the claim in (5.1).

The next proposition also follows with similar arguments.

Proposition 5.2. Let $y_{1}, y_{2}$ be such that $u\left(y_{1}\right)=u\left(y_{2}\right)=1$ with $y_{1} \cdot e_{1}>y_{2} \cdot e_{1}$, and let $v$ be the direction perpendicular to $y_{1}-y_{2}$ with $v_{2}>0$. If

$$
l_{\sigma}\left(y_{1}, y_{2}\right):=\left\{x \mid\left(x-y_{1}\right) \cdot v=\sigma\right\} \cap\left\{x \mid x \cdot e_{1} \in\left(y_{1} \cdot e_{1}, y_{2} \cdot e_{1}\right)\right\},
$$

then

$$
l_{\sigma} \subset\{u>\sigma\} .
$$

Proof. Consider the linear one-dimensional solution $v$ to $F\left(D^{2} v\right)=0$ and $|\nabla v|=$ 1 in $\{0<v<1\}$ which is equal to 0 on the line $l_{0}$ connecting $y_{1}$ and $y_{2}$ and increases in the $e_{2}$ direction. Denote by $l_{1}$ the 1 level set of $v$ and by $z_{1}, z_{2}$ the intersection points of the vertical segments through $y_{1}, y_{2}$ with $l_{1}$. Also, let $D$ be the open parallelogram with vertices at $y_{i}, z_{i}, i=1,2$.

It suffices to show that

$$
u>v \text { in } D
$$


since

$$
l_{\sigma}\left(y_{1}, y_{2}\right) \subset D
$$

and

$$
v=\sigma \quad \text { on } l_{\sigma}\left(y_{1}, y_{2}\right) .
$$

First let us notice that since $u=1$ above $\mathcal{J}_{1}$ then

$$
\begin{gathered}
u>v \text { on }\left[y_{i}, z_{i}\right), \\
z_{i} \quad \text { is strictly above } \mathcal{J}_{1} .
\end{gathered}
$$

Now let us apply the sliding method (upside-down). Since $u=1$ above $\mathcal{J}_{1}$, there exists $t_{0}>0$ such that $u$ is above $v_{t}$ for all $t \geq t_{0}$. Let $\bar{t}$ be the largest $t \leq t_{0}$ such that $u$ touches $v_{t}$ from above. Assume by contradiction that $\bar{t} \geq 0$. Again,

$$
\begin{gathered}
u>v_{\bar{t}} \text { on }\left[y_{i}+\bar{t} e_{2}, z_{i}+\bar{t} e_{2}\right), \\
z_{i}+\bar{t} e_{2} \quad \text { is strictly above } \mathcal{J}_{1}
\end{gathered}
$$

In particular $u$ and $v_{\bar{t}}$ cannot coincide. Thus, similarly to the proof of Proposition 5.1, the comparison principle, Hopf lemma and (5.9) imply that

$$
\begin{gathered}
u>v_{\bar{t}} \text { on }\left(\bar{D}+\bar{t} e_{2}\right) \backslash\left[z_{1}+\bar{t} e_{2}, z_{2}+\bar{t} e_{2}\right], \\
{\left[z_{1}+\bar{t} e_{2}, z_{2}+\bar{t} e_{2}\right] \Subset\{u=1\} .}
\end{gathered}
$$

Thus for small $\varepsilon>0, u$ is above $v_{\bar{t}-\varepsilon}$ which contradicts the definition of $\bar{t}$. Hence $\bar{t}<0$ and $u$ is above $v=v_{0}$ in $\bar{D}$. In particular, $u>v$ on $\bar{D} \backslash\left[z_{1}, z_{2}\right]$ and hence $u>\sigma$ on the $\sigma$ level set $l_{\sigma}\left(y_{1}, y_{2}\right)$ of $v$.

Now we are ready to show that $\{u=\sigma\}$ is included in a strip. We start by defining $E_{R, \sigma}$ to be the convex hull generated by $A_{R, \sigma}$.

From Proposition 5.1, we have that

$$
E_{R, \sigma} \subset\{u<\sigma\}
$$

As a consequence of Lemma 4.3(ii), we obtain the following lemma.

Lemma 5.3. There exists $C$ large universal constant such that in any vertical strip of width $2 C,\left\{a-C \leq x_{1} \leq a+C\right\}$ there exists a point on $\partial E_{C, \sigma}$ that is at distance less than $2 C$ from $\mathcal{J}_{1}$.

Proof. Let $B_{C}(y)$ be the ball with center on the line $x_{1}=a$ which is tangent to $\{u=\sigma\}$ at $x_{0}$, with $y \in A_{C, \sigma}$. By Lemma 4.3 there exists

$$
x_{1} \in \mathcal{J}_{1} \cap B_{K}\left(x_{0}\right) .
$$

Then the desired point is the intersection of $\left[x_{1}, y\right]$ with $\partial E_{C, \sigma}$, provided that $C>K$. 
Lemma 5.4. If $C$ is large enough, the set $E:=E_{C, \sigma}$ is a half-plane.

Proof. Since $E$ is a convex set and for any $x_{0} \in \mathbb{R}^{2}$ there exists $t_{0}$ such that $x_{0}+$ $t e_{2} \in E$ for all $t \leq t_{0}$, then $\partial E$ is the graph of a concave function $f$ i.e.

$$
\partial E=\{(s, f(s)), s \in \mathbb{R}\} .
$$

If $f$ is not linear, we can find a linear function $p(s)$ tangent to $f$ at some $s_{0}$ such that

$$
\lim _{s \rightarrow \pm \infty}(p(s)-f(s))=+\infty .
$$

From Lemma 5.3 for each $n \in \mathbb{N}$ there exist points

$$
\begin{aligned}
& y_{n} \in\left\{x \mid(n-3) C \leq x \cdot e_{1} \leq(n+3) C\right\} \cap \mathcal{J}_{1} \\
& z_{n} \in\left\{x \mid-(n+3) C \leq x \cdot e_{1} \leq-(n-3) C\right\} \cap \mathcal{J}_{1}
\end{aligned}
$$

that are at distance at most $2 C$ from the graph of $f$.

From (5.11) we see that as $n \rightarrow \infty$, the point $\left(s_{0}, f\left(s_{0}\right)\right)$ is at an arbitrary large distance above the line passing through $y_{n}, z_{n}$.

Thus, when this distance becomes greater than $2 \sigma$, in the light of (5.8), we can find a point

$$
\left(s_{0}, t\right) \in l_{\sigma}\left(y_{n}, z_{n}\right), \quad \text { for some } t<f\left(s_{0}\right) .
$$

From Proposition 5.2 we know that

$$
\left(s_{0}, t\right) \in\{u>\sigma\} .
$$

On the other hand, since $t<f\left(s_{0}\right)$,

$$
\left(s_{0}, t\right) \in E \subset\{u<\sigma\} .
$$

Thus we reached a contradiction. This shows that $f$ is a linear function.

Corollary 5.5. $\{u=\sigma\}$ is contained in a strip. The direction $v$ of the strip satisfies $\nu_{2} \neq 0$. Furthermore, $\Omega$ is also contained in a strip in the $v$ direction.

Proof. From (5.10), Lemma 5.4 (and their counterpart in $\{u>\sigma\}$ ), we conclude that $\{u=\sigma\}$ is contained in a strip, say $\{a \leq x \cdot v \leq b\}$. Also, $v_{2} \neq 0$ since $\{u=\sigma\}$ is trapped between $\mathcal{J}_{0}$ and $\mathcal{J}_{1}$ which are graphs in the $e_{2}$ direction.

Now, applying Lemma 4.3(i) together with its counterpart in $\{u>\sigma\}$, with $R$ a fixed large constant, we conclude that $\mathcal{J}_{0}$ is above the line $\{x \cdot v=a-C\}$ while $\mathcal{J}_{1}$ is below the line $\{x \cdot v=b+C\}$, for some $C$ universal. This concludes the proof of the corollary.

Next, we show that the width of the strip obtained in Corollary 5.5 is arbitrarily small and hence $\{u=\sigma\}$ is a line, which concludes the proof of Theorem 1.1. 
As a preliminary step in this direction, we prove that $u$ is monotone in the vertical direction, so to be able to apply our Harnack inequality (this is also a nice consequence of Corollary 5.5).

Proposition 5.6. $u$ is monotone increasing in the $e_{2}$ direction.

Proof. Assume for simplicity that $u \in C^{3}(\Omega)$ and $F \in C^{1}$.

From Corollary 5.5 we have that $\Omega$ is included in a strip, say

$$
S:=\{-a \leq x \cdot v \leq a\}
$$

Notice that

$$
u_{2}:=\frac{\partial u}{\partial x_{2}} \geq 0 \quad \text { near } \partial \Omega
$$

and by Lipschitz continuity (see Lemma 4.1)

$$
u_{2} \geq-K \quad \text { in } \Omega
$$

with $K>0$ universal. Also, $u_{2}$ solves the linearized equation

$$
a_{i j}(x)\left(u_{2}\right)_{i j}=0 \quad \text { in } \Omega
$$

with

$$
a_{i j}(x)=F_{i j}\left(D^{2} u\right)
$$

We compare $u_{2}$ with $W(x)=w\left(x \cdot v^{\perp}, x \cdot v\right)$ in the domain $\Omega \cap\left\{\left|x \cdot v^{\perp}\right|<L\right\}$, where

$$
w(s, t)=K a^{-2} e^{-L \delta}\left(e^{-\delta s}+e^{\delta s}\right)\left(t^{2}-2 a^{2}\right) .
$$

Here $L>0$ is fixed and it will be taken large in the sequel.

Notice that

$$
W \leq 0 \text { in } S
$$

and also

$$
\lambda\left|\left(D^{2} w\right)^{+}\right|-\Lambda\left|\left(D^{2} w\right)^{-}\right|>0
$$

if $\delta$ is small depending on $\lambda, \Lambda$.

This implies that

$$
a_{i j}(x) W_{i j}(x)>0 .
$$

Since

$$
W \leq 0 \text { on } \partial \Omega
$$

due to (5.12), and

$$
W \leq-K \quad \text { in } S \cap\left\{\left|x \cdot v^{\perp}\right|=L\right\}
$$


we conclude from the maximum principle that

$$
u_{2} \geq W \text { in } \Omega \cap\left\{\left|x \cdot v^{\perp}\right|<L\right\} .
$$

On $\left\{\left|x \cdot v^{\perp}\right| \leq L / 2\right\} \cap \Omega$ we obtain that $u_{2} \geq-4 K e^{-3 \delta L / 2}$ and by letting $L \rightarrow \infty$ we obtain that in fact $u_{2} \geq 0$ in $\Omega$.

The smoothness assumptions on $u$ and $F$ can be removed with the techniques of [2].

Now, we are ready to complete the proof of our main theorem.

Proof of Theorem 1.1. The idea of the proof is that once $\{u=\sigma\}$ is included in some strip of width, say, $d>0$, then it is included in a smaller strip, say of width $3 d / 4$. From this, of course, it would follow that $\{u=\sigma\}$ is a line.

Here are the details of such an improvement of flatness.

By Corollary 5.5, we may assume that

$$
\{u=\sigma\} \subset\{0<x \cdot v<d\},
$$

for some direction $v$ with $v_{2}>0$.

Let $B_{R}\left(x_{*}\right)$ be a large ball with center on the line $t v$ which touches $\{u=\sigma\}$ from below at $x_{0}$. Let $v^{*}=\left(x_{0}-x_{*}\right) / R$.

We distinguish two cases.

Case 1. $x_{0} \cdot v>d / 2$. In this case the point

$$
y_{0}:=x_{0}-\frac{2 C}{R} v^{*}
$$

with $C$ as in Proposition 5.1 satisfies

$$
y_{0} \cdot v>\frac{d}{4}
$$

as long as $R$ is large enough.

By applying Proposition 5.1 to the ball $B_{R}\left(x_{*}\right)$ and all the balls of radius $R$ tangent from below to $\{x \cdot v=0\}$, which lie in $\{u<\sigma\}$ because of (5.13), we find that $\{u=\sigma\}$ is above the line $\left\{x \cdot v=y_{0}\right\}$.

So, by (5.14),

$$
\{u=\sigma\} \text { is above the line }\{x \cdot v=d / 4\} .
$$

Case 2. $x_{0} \cdot v \leq d / 2$. In this case, the point

$$
y_{d}:=x_{0}+\frac{\bar{C}}{R} v^{*}+\frac{2 C}{M-\bar{C} / R} v^{*}
$$


satisfies

$$
y_{d} \cdot v<\frac{3 d}{4}
$$

as long as $R$ and $M$ are large (again $C$ is as in Proposition 5.1). Notice also that $y_{d}$ is in the ball of radius

$$
M-\frac{\bar{C}}{R}-\frac{C}{M-\bar{C} / R}
$$

centered at $\left(M v^{*}+x_{0}\right)$.

Also, from Lemma 4.4,

$$
B_{M-\frac{\bar{C}}{R}}\left(M v^{*}+x_{0}\right) \subset\{u>\sigma\}
$$

if $R$ is large.

Therefore, by applying Proposition 5.1 (upside-down) to the ball $B_{M-\frac{\bar{C}}{R}}\left(M v^{*}+\right.$ $\left.x_{0}\right)$ and all the balls of the same radius $R$ tangent from above to $\{x \cdot v=d\}$ we find that $\{u=\sigma\}$ is below the line $\left\{x \cdot v=y_{d} \cdot v\right\}$.

This and (5.16) give that

$$
\{u=\sigma\} \text { is below the line }\{x \cdot v=3 d / 4\} .
$$

In either cases, from (5.13) and either (5.15) or (5.17), we obtain that $\{u=\sigma\}$ is included in a strip of width $3 d / 4$, which is the desired improvement of flatness.

\section{References}

[1] H. W. Alt and L. A. CAFFARELli, Existence and regularity for a minimum problem with free boundary, J. Reine Angew. Math. 325 (1981), 105-144.

[2] L. A. CAfFARelli and X. CABrÉ, "Fully Nonlinear Elliptic Equations", Vol. 43 of American Mathematical Society Colloquium Publications, American Mathematical Society, Providence, RI, 1995.

[3] L. A. CAFfarelli and S. SAlsa, "A Geometric Approach to Free Boundary Problems", GSM 68, American Mathematical Society, Providence, Rhode Island, 2005.

[4] D. DE SILVA and O. SAVIn, Symmetry of global solutions to a class of fully nonlinear elliptic equations in 2D, Indiana Univ. Math. J. 58 (2009), 301-315.

[5] J. Dolbeault and R. Monneau, On a Liouville type theorem for isotropic homogeneous fully nonlinear elliptic equations in dimension two, Ann. Scuola Norm. Sup. Pisa Cl. Sci. (5) 2 (2003), 181-197.

[6] A. FARINA and E. VALDINOCI, Flattening results for elliptic PDEs in unbounded domains with applications to overdetermined problems, Arch. Ration. Mech. Anal. 195 (2010), $1025-1058$.

[7] O. SAVIn, "Phase Transitions: Regularity of Flat Level Sets", PhD thesis, University of Texas at Austin, 2003.

[8] O. SAVIn, Entire solutions to a class of fully nonlinear elliptic equations, Ann. Scuola Norm. Sup. Pisa Cl. Sci. (5) 3 (2008), 369-405.

[9] O. SAVIN, Regularity of flat sets in phase transitions, Ann. of Math. 169 (2009), 41-78. 
[10] E. VALDINOCI, Bernoulli jets and the zero mean curvature equation, J. Differential Equations 225 (2006), 710-736.

[11] E. VALdinOCI, Flatness of Bernoulli jets, Math. Z. 254 (2006), 257-298.

Department of Mathematics

Barnard College

Columbia University

NY 10027, New York

desilva@math.columbia.edu

Dipartimento di Matematica

II Università di Roma "Tor Vergata"

Via della Ricerca Scientifica

00133 Roma, Italia

enrico.valdinoci@uniroma2.it 